\title{
CTNNA3 genetic polymorphism may be a new genetic signal of type 2 diabetes in the Chinese Han population: a case control study
}

Yunjun Zhang ${ }^{1 \dagger}$, Xiaoman Zhou ${ }^{1 \dagger}$, Wanjuan Dai ${ }^{2}$, Juan Sun ${ }^{1}$, Mei Lin ${ }^{1}$, Yutian Zhang $^{1}$ and Yipeng Ding ${ }^{1 *}$

\begin{abstract}
Background: Type 2 Diabetes (T2D) is the result of a combination of genes and environment. The identified genetic loci can only explain part of T2D risk. Our study is aimed to explore the association between CTNNA3 single nucleotide polymorphisms (SNPS) and T2D risk.

Methods: We conducted a 'case-control' study among 1002 Chinese Han participants. Four candidate SNPs of CTNNA3 were selected (rs10822745 C/T, rs7920624 A/T, rs2441727 A/G, rs7914287 A/G), and logistic regression analysis was used to evaluate the association between candidate SNPs and T2D risk. We used single factor analysis of variance to analyze the differences of clinical characteristics among different genotypes. In this study, haplotype analysis was conducted by plink1.07 and Haploview software and linkage disequilibrium (LD) was calculated. The interaction of candidate SNPs in T2D risk was evaluated by multi-factor dimensionality reduction (MDR). Finally, we conducted a false-positive report probability (FPRP) analysis to detect whether the significant findings were just chance or noteworthy observations.
\end{abstract}

Results: The results showed that CTNNA3-rs7914287 was a risk factor for T2D ('T': OR=1.33, $p=0.003 ;$ 'TT': OR=2.21, $p=0.001 ; ' T^{\prime}$ ' (recessive): $\mathrm{OR}=2.09, p=0.001$; Log-additive: $\mathrm{OR}=1.34, p=0.003$ ). The results of subgroup analysis showed that rs 7914287 was significantly associated with the increased risk of T2D among participants who were older than 60 years, males, smoking, drinking, or BMI > 24. We also found that rs 2441727 was associated with reducing the T2D risk among participants who were older than 60 years, smoking, or drinking. In addition, rs7914287 was associated with T2D patients with no retinal degeneration; rs 10822745 and rs7920624 were associated with the course of T2D patients. High density lipoprotein levels had significant differences under different genotypes of rs 10822745. Under the different genotypes of rs 7914287 , the levels of aspartate aminotransferase, alanine aminotransferase and gamma-glutamyltransferase were also significantly different.

Conclusion: We found that CTNNA3 genetic polymorphisms can be used as a new genetic signal of T2D risk in Chinese Han population. Especially, CTNNA3-rs7914287 showed an outstanding and significant association with T2D risk in both overall analysis and subgroup analysis.

\footnotetext{
*Correspondence: ypding1961@163.com

${ }^{\dagger}$ Yunjun Zhang and Xiaoman Zhou are co-first authors

1 Department of General Practice, Hainan General Hospital, \#19, Xiuhua

Road, Xiuying District, Haikou 570311, Hainan, People's Republic of China

Full list of author information is available at the end of the article
}

(c) The Author(s) 2021. Open Access This article is licensed under a Creative Commons Attribution 4.0 International License, which permits use, sharing, adaptation, distribution and reproduction in any medium or format, as long as you give appropriate credit to the original author(s) and the source, provide a link to the Creative Commons licence, and indicate if changes were made. The images or other third party material in this article are included in the article's Creative Commons licence, unless indicated otherwise in a credit line to the material. If material is not included in the article's Creative Commons licence and your intended use is not permitted by statutory regulation or exceeds the permitted use, you will need to obtain permission directly from the copyright holder. To view a copy of this licence, visit http://creativecommons.org/licenses/by/4.0/. The Creative Commons Public Domain Dedication waiver (http://creativeco mmons.org/publicdomain/zero/1.0/) applies to the data made available in this article, unless otherwise stated in a credit line to the data. 
Keywords: Type 2 diabetes, Case-control study, CTNNA3, Single nucleotide polymorphisms

\section{Introduction}

Type 2 diabetes (T2D) is non-insulin-dependent diabetes, is a form of diabetes is much more prevalent.The prevalence of T2D is getting higher and higher worldwide. T2D and its complications have reached the level of an epidemic, which has attracted great attention in the field of scientific research [1]. A number of studies have confirmed that T2D is a complex disease affected by multiple factors and is the result of the interaction between genes and the environment [1-4]. Evidence from genetic epidemiology indicates that the occurrence of type 2 diabetes has a strong genetic basis [5-8]. In recent years, with the improvement and application of molecular epidemiology or genetic testing technology, some T2D-related genetic loci have been identified one after another [9-15], but it can only explain the T2D risk among part of population $[1,16]$. Therefore, it is necessary to discover new genetic signals of T2D in different populations, which will provide valuable references for clinical diagnosis and early prevention of T2D.

CTNNA3 can encode $\alpha$ T-catenin protein and is a member of the $\alpha$-catenin family of cell-cell adhesion molecules [17]. Chiarella, S. E., et al. have proposed that CTNNA3 may be the most relevant type of $\alpha$-catenin in human diseases [18]. In a recent genome-wide association study on Metabolic Syndrome of African Lineage (MetS), single nucleotide polymorphism (SNPs) of CTNNA3 were reported can be new genetic signals of metabolic syndrome risk. There have been studies have reported that people with MetS have at least a five-fold increase in the risk of T2D $[19,20]$. But so far, there is no report about the association between CTNNA3 SNPs and T2D risk.

Therefore, in order to explore the association between CTNNA3 SNPs and T2D risk, we conducted a 'case-control' study among a total of 1,002 Chinese Han population. We not only conducted an overall analysis, but also divided participants according to the known potential environmental risk factors of T2D for subgroup analysis, such as drinking [21], smoking [22], age [23], etc. Finally, the association between candidate SNPs and T2D risk will be evaluated. Our study will provide data supplement for genetic loci associated with T2D risk in Chinese Han population, and lay a certain theoretical foundation for the individualized prevention and treatment of T2D.

\section{Materials and methods Study subjects}

In this study, a 'case-control' study design was used to analyze the association between SNPs and T2D risk among 1002 participants (501 cases and 501 controls). Case group: (1) T2D patients who are outpatients or hospitalized in Hainan General Hospital. (2) Patients who were diagnosed as T2D for the first time or who have been clearly diagnosed as T2D (Fasting blood glucose (FBG) $\geq 7.0 \mathrm{mmol} / \mathrm{L}$, OGTT $2 \mathrm{~h}$ blood glucose $\geq 11.1 \mathrm{mmol} / \mathrm{L}$ or random blood glucose $\geq 11.1 \mathrm{mmol} / \mathrm{L}$ ). (3) No history of other complicated diseases (malignant tumors, cardiovascular disease history, etc.). (4) No history of genetic diseases. Control group: (1) Healthy individuals undergoing physical examination at the same hospital's health examination center during the same period. (2) FBG $\leq 6.1 \mathrm{mmol} / \mathrm{L}$. (3) No complicated chronic diseases, and tumor patients or people with tumor history are excluded. (4) Recruit healthy individuals who match the case group in terms of age and gender (excluding confounding factors caused by differences in the distribution of exposure factors in the case/ control group).

In this study, questionnaire survey on demographic and epidemiological information among all participants was conducted by a professional doctor. The contents of the questionnaire include gender, height, weight, smoking/ drinking status, whether diabetic patients were accompanied by retinopathy, and the course of T2D. After obtaining the informed consent of all participants, we collected their peripheral blood samples for subsequent DNA extraction. The study was approved by the ethics committee of Hainan General Hospital.

\section{Selection of SNPs}

The specific steps for selecting SNPs are as follows: (1) We obtained the physical position of the CTNNA3 on the Chromosome 10: 65,912,457-67,763,637 through the e!GRCh37 database (http://asia.ensembl.org/Homo_sapie ns/Info/Index). In the VCF to PED Converter window (http://grch37.ensembl.org/Homo_sapiens/Tools/Vcfto Ped), we entered the gene location, selected the CHB and CHS population, and downloaded the ped and info file for the variations of CTNNA3. (2) Then we used Haploview software for quality control (HWE $>0.01, \mathrm{MAF}>0.05$, Min Genotype $>75 \%$, and Tagger $\left.\mathrm{r}^{2}>0.8\right)$ to select tagSNP. Finally, four SNPs of CTNNA3 (rs10822745 C/T, rs7920624 A/T, rs2441727 A/G, rs7914287 A/G) were selected for our study. You can check the revisions from the 'Methods-Selection of SNPs.

\section{DNA extraction and genotyping}

DNA extraction and purification were performed according to the instructions of the kit (GoldMag Co. Ltd. Xi'an, 
China). We store the purified DNA in an ultra-low temperature refrigerator $\left(-80{ }^{\circ} \mathrm{C}\right)$ until needed in the next experiment. We used MassARRAY Assay Design software to design all primers we need. The genotyping in this study was conducted by the MassARRAY system (Agena, San Diego, CA, USA).

In order to reduce experimental errors and ensure the reliability and repeatability of experimental results, we randomly select $10 \%$ of DNA samples for repeatability testing. The repetition rate of experimental results needs to be $>99 \%$.

\section{Statistical analysis}

The differences in demographic characteristics (age, gender, BMI, etc.) were tested by SPSS version 21.0 software (SPSS, Chicago, IL, USA) ( $\chi^{2}$ test $/ t$-test). After testing whether the four candidate genetic loci of CTNNA3 meet Hardy-Weinberg equilibrium (SPSS version 21.0 software), we used logistic regression model to calculate the odds ratio (OR) and 95\% confidence interval $(\mathrm{CI})$. Then, according to the value of OR and $\mathrm{CI}$, the association between CTNNA3 candidate SNPs and T2D risk was estimated (OR value represents relative risk; $\mathrm{OR}=1$ : this factor has no effect on $\mathrm{T} 2 \mathrm{D}$ risk; $\mathrm{OR}<1$ : $\mathrm{T} 2 \mathrm{D}$ protective factor; $\mathrm{OR}>1$ : $\mathrm{T} 2 \mathrm{D}$ risk factor). Using the wild-type allele as a reference, the online tool software plink 1.07 was used to estimate multiple genetic models. The statistical results obtained were adjusted by age and gender, and all tests were twosided tests. In addition, we conducted a false-positive report probability (FPRP) analysis to detect whether the significant findings were just chance or noteworthy observations [24]. In this study, haplotype analysis was conducted by plink 1.07 and Haploview software and linkage disequilibrium (LD) was calculated. Finally, the interaction of candidate SNPs in T2D risk was evaluated by multi-factor dimensionality reduction (MDR).

\section{Results}

\section{Sample overview and collection}

There was no genetic relationship among all participants in our study. Among them, the average age of T2D patients was $59.86 \pm 12.86$ years old, males 359 (72\%), females $142(28 \%)$. The average age of healthy individuals was $59.60 \pm 10.09$ years, males $359(72 \%)$, females 142 (28\%). The basic demographic and epidemiological information was shown in Table 1 . There was no statistical difference between the case and the control group in gender $(p=0.528)$ or age $(p=0.714)$.

\section{Genotyping and information about candidate SNPs}

The 4 candidate genetic loci of CNNTA3 (rs10822745 $\mathrm{C} / \mathrm{T}, \mathrm{rs} 7920624 \mathrm{~A} / \mathrm{T}, \mathrm{rs} 2441727 \mathrm{~A} / \mathrm{G}, \mathrm{rs} 7914287 \mathrm{~A} / \mathrm{G}$ ) were successfully genotyped. As shown in Table 2, all candidate SNPs met HWE ( $p>5 \%)$. The results of HaploReg showed that the candidate SNPs were regulated by a variety of factors, and the specific factors were detailed in Table 2.

Table 1 Characteristics of patients with type 2 diabetes and healthy individuals

\begin{tabular}{|c|c|c|c|c|}
\hline \multicolumn{2}{|l|}{ Characteristics } & $\begin{array}{l}\text { Cases } \\
\mathrm{n}=501\end{array}$ & \multirow{2}{*}{$\begin{array}{l}\begin{array}{l}\text { Control } \\
\mathbf{n}=\mathbf{5 0 1}\end{array} \\
59.60 \pm 10.09\end{array}$} & $P$ \\
\hline \multirow[t]{3}{*}{ Age (years) } & Mean $\pm S D$ & $59.86 \pm 12.86$ & & 0.714 \\
\hline & $>60$ & $240(48 \%)$ & $262(52 \%)$ & \\
\hline & $\leq 60$ & $261(52 \%)$ & $239(48 \%)$ & \\
\hline \multirow[t]{2}{*}{ Gender } & Male & $359(72 \%)$ & $359(72 \%)$ & 0.528 \\
\hline & Female & $142(28 \%)$ & $142(28 \%)$ & \\
\hline Course of disease & $>10$ versus $\leq 10$ years & $194(39 \%)$ & $306(61 \%)$ & \\
\hline \multirow[t]{2}{*}{ No retinal degeneration } & Yes & 70 (14\%) & - & \\
\hline & No & $240(48 \%)$ & - & \\
\hline \multirow[t]{2}{*}{ Smoking } & Yes & $218(44 \%)$ & $124(25 \%)$ & 0.593 \\
\hline & No & $281(56 \%)$ & $173(35 \%)$ & \\
\hline \multirow[t]{2}{*}{ Drinking } & Yes & 109 (22\%) & $127(25 \%)$ & $<0.0001$ \\
\hline & No & $385(77 \%)$ & $143(29 \%)$ & \\
\hline \multirow[t]{2}{*}{$\mathrm{BMI}\left(\mathrm{kg} / \mathrm{m}^{2}\right)$} & $\mathrm{BMI}>24$ & $239(48 \%)$ & 187 (37\%) & 0.089 \\
\hline & $\mathrm{BMI} \leq 24$ & $203(41 \%)$ & $123(25 \%)$ & \\
\hline
\end{tabular}

Course of disease: the length of time the case has suffered from T2D (participants are divided by the average of the length of time) 
Table 2 The basic information and HWE about the selected SNPs of CTNNA3

\begin{tabular}{|c|c|c|c|c|c|c|c|c|}
\hline \multirow[t]{2}{*}{ Gene } & \multirow[t]{2}{*}{ SNP ID } & \multirow[t]{2}{*}{ Function } & \multirow[t]{2}{*}{ Chr: position } & \multirow{2}{*}{$\begin{array}{l}\text { Alleles } \\
\text { (A/B) }\end{array}$} & \multicolumn{2}{|l|}{ MAF } & \multirow{2}{*}{$\begin{array}{l}\text { HWE } \\
\text { ( } P \text { value })\end{array}$} & \multirow[t]{2}{*}{ Haploreg 4.1} \\
\hline & & & & & Cases & Controls & & \\
\hline CTNNA3 & rs10822745 & Intronic & 10: 66,194,307 & $\mathrm{C} / \mathrm{T}$ & 0.429 & 0.417 & 0.646 & Motifs changed \\
\hline CTNNA3 & rs7920624 & Intronic & $10: 66,203,428$ & $\mathrm{~A} / \mathrm{T}$ & 0.488 & 0.498 & 0.929 & Motifs changed \\
\hline CTNNA3 & rs2441727 & Intronic & 10: 66,465,128 & $A / G$ & 0.182 & 0.204 & 0.406 & NHGRI/EBI GWAS hits \\
\hline CTNNA3 & rs7914287 & Intronic & $10: 67,590,805$ & $\mathrm{~T} / \mathrm{C}$ & 0.355 & 0.292 & 0.124 & - \\
\hline
\end{tabular}

HWE, Hardy-Weinberg equilibrium; Alleles (A/B), minor/major allele; SNP, single nucleotide polymorphisms; MAF, minor allele frequency $p>0.05$ indicates that the genotypes were in Hardy-Weinberg Equilibrium

\section{Evaluation of association between candidate SNPs and T2D risk (overall analysis)}

The evaluation results of the association between candidate SNPs and T2D risk (Table 3) showed that only CNNTA3 rs7914287 had a significant association with the T2D risk among participants. Specifically, rs7914287 can significantly increase the T2D risk under allele ( $\mathrm{T}$ vs. $\mathrm{C}: \mathrm{OR}=1.33$, CI 1.10-1.61, $p=0.003$ ), homozygous (TT vs. $\mathrm{CC}: \mathrm{OR}=2.21, \mathrm{CI} 1.41-3.47, p=0.001$ ), recessive (TT vs. TC-CC: $\mathrm{OR}=2.09, \mathrm{CI} 1.36-3.22, p=0.001)$, and logadditive models ( $\mathrm{OR}=1.34$, CI 1.10-1.62, $p=0.003)$. We did not find any evidence that the remaining three candidate SNPs were associated with the T2D risk.

\section{Evaluation of association between candidate SNPs and T2D risk (subgroup analysis)}

Age and gender The results showed (Table 4) that CNNTA3 rs2441727 can significantly reduce the T2D risk in participants who were aged $\leq 60$ years old under multiple genetic models (heterozygote: $\mathrm{OR}=0.58$, CI0.39-0.87, $p=0.008$; dominant: $\mathrm{OR}=0.64$, CI 0.440.93, $p=0.021$ ). rs7914287 was not only a risk factor for T2D in participants aged $>60$ years old (allele: $\mathrm{OR}=1.37$, CI 1.06-1.79, $p=0.018$; homozygote: $\mathrm{OR}=2.22$, CI 1.18$4.17, p=0.013$; dominant: $\mathrm{OR}=1.56$, CI 1.08-2.25, $p=0.019$; recessive: $\mathrm{OR}=1.85$, CI $1.02-3.35, p=0.044$; log-additive: $\mathrm{OR}=1.47, \mathrm{CI} 1.11-1.94, p=0.007)$, but also a risk factor for T2D in participants aged $\leq 60$ years old (homozygote: $\mathrm{OR}=2.71, \mathrm{CI} 1.36-5.42, p=0.005$; recessive: $\mathrm{OR}=2.73, \mathrm{CI} 1.40-5.32, \quad p=0.003$; log-additive: $\mathrm{OR}=1.33$, CI 1.01-1.75, $p=0.041$ ). In the gender stratification analysis, rs7914287 can significantly increase the T2D risk in male participants (Allele: $\mathrm{OR}=2.33$, CI 1.073.67, $p=0.012$; homozygote: $\mathrm{OR}=2.20, \mathrm{CI} 1.29-3.77$, $p=0.004$; recessive: $\mathrm{OR}=2.08, \mathrm{CI} 1.24-3.49, p=0.005$; log-additive: $\mathrm{OR}=2.33$, CI 1.06-3.67, $p=0.013$ ).

Smoking and drinking The results showed (Table 5) that rs2441727 significantly reduced the T2D risk in participants with a history of smoking (heterozygote: $\mathrm{OR}=0.61$, CI0.41-0.92, $p=0.018$; dominant: $\mathrm{OR}=0.65$, CI0.44$0.97, p=0.034)$. At the same time, rs2441727 reduced the T2D risk in drinking participants under the heterozygous genetic model (heterozygote: $\mathrm{OR}=0.63$, CI0.420.94, $p=0.025)$. rs7914287 was a risk factor for T2D in smoking (allele: $\mathrm{OR}=2.36, \mathrm{CI} 1.01-4.82, p=0.023$; homozygote: $\mathrm{OR}=2.50, \mathrm{CI} 1.18-5.32, p=0.017$; recessive: $\mathrm{OR}=2.44, \mathrm{CI} 1.18-5.05, p=0.016)$ and drinking participants (allele: $\mathrm{OR}=1.41, \mathrm{CI} 1.04-2.91, p=0.025$; homozygote: $\mathrm{OR}=2.11, \mathrm{CI} 1.34-5.20, p=0.008$; recessive: $\mathrm{OR}=2.00$, CI 1.33-4.77, $p=0.008$ ).

$B M I$ The results showed (Table 6) that rs7914287 was a risk factor for T2D no matter in the participants with $\mathrm{BMI} \leq 24$ or $\mathrm{BMI}>24$. Specifically, among the participants with $\mathrm{BMI} \leq 24$, rs7914287 can significantly increase the T2D risk under allele $(\mathrm{OR}=1.57, \mathrm{CI} 1.11$ 2.24, $p=0.011)$, homozygote $(\mathrm{OR}=3.66, \mathrm{CI} 1.43-5.36$, $p=0.007)$, recessive ( $\mathrm{OR}=2.25, \mathrm{CI} 1.31-4.07, p=0.011)$, and log-additive genetic models $(\mathrm{OR}=1.60, \mathrm{CI} 1.12-$ 2.29, $p=0.010$ ). Among the participants with $\mathrm{BMI}>24$, rs7914287 can also significantly increase the T2D risk under allele $(\mathrm{OR}=1.45, \mathrm{CI} 1.08-1.96, p=0.014)$, homozygote $(\mathrm{OR}=2.86$, CI 1.32-6.24, $p=0.008)$, recessive $(\mathrm{OR}=2.58$, CI 1.22-4.47, $p=0.014)$, and log-additive genetic models (OR $=1.48, \mathrm{CI} 1.09-2.01, p=0.013)$.

No retinal degeneration and course of T2D The results showed (Table 7) that rs7914287 was associated with T2D patients who have no retinal egeneration under multiple genetic models (allele: $p=0.022$, homozygote: $p=0.004$, recessive: $p=0.003$, log-additive: $p=0.021$ ). We also found that the candidate SNPs associated with the course of T2D were rs10822745 (allele: $p=0.022$, homozygote: $p=0.017$, recessive: $p=0.027$, log-additive: $p=0.023$ ), rs7920624 (allele: $p=0.030$ ), and rs2441727 (allele: $p=0.030$; heterozygote: $p=0.001$; dominant: $p=0.001$; log-additive: $p=0.003$ ).

Differences in clinical indicators under different genotypes We also evaluated the impact of 4 candidate CTNNA3 SNPs on the level of clinical indicators under different genotypes. The result showed (Table 8) that high density lipoprotein levels had significant differences under different genotypes of CTNNA3 rs10822745 
Table 3 Analysis of the association between susceptibility of type 2 diabetes and single nucleotide polymorphism of CTNNA3

\begin{tabular}{|c|c|c|c|c|c|c|}
\hline \multirow[t]{2}{*}{ SNP ID } & \multirow[t]{2}{*}{ Model } & \multirow[t]{2}{*}{ Genotype } & \multirow[t]{2}{*}{ Case } & \multirow[t]{2}{*}{ Control } & \multicolumn{2}{|c|}{ Adjusted by age and gender } \\
\hline & & & & & OR $(95 \% \mathrm{Cl})$ & $p$ \\
\hline \multirow[t]{10}{*}{ rs10822745 } & \multirow[t]{2}{*}{ Allele } & C & 430 (42.91\%) & 417 (41.70\%) & $1.05(0.88-1.26)$ & 0.582 \\
\hline & & $\mathrm{T}$ & 572 (57.09\%) & 583 (58.30\%) & 1.00 & \\
\hline & \multirow[t]{3}{*}{ Genotype } & $\mathrm{CC}$ & $87(17.4 \%)$ & 84 (16.8\%) & $1.10(0.76-1.59)$ & 0.631 \\
\hline & & $\mathrm{CT}$ & $256(51.1 \%)$ & $249(49.8 \%)$ & $1.09(0.82-1.44)$ & 0.558 \\
\hline & & $\mathrm{TT}$ & $158(31.5 \%)$ & $167(33.4 \%)$ & 1.00 & \\
\hline & \multirow[t]{2}{*}{ Dominant } & $\mathrm{CC}-\mathrm{CT}$ & $343(68.5 \%)$ & $333(66.6 \%)$ & $1.09(0.84-1.42)$ & 0.528 \\
\hline & & $\mathrm{TT}$ & $158(31.5 \%)$ & $167(33.4 \%)$ & 1.00 & \\
\hline & \multirow[t]{2}{*}{ Recessive } & $\mathrm{CC}$ & 87 (17.4\%) & $84(16.8 \%)$ & $1.04(0.75-1.45)$ & 0.811 \\
\hline & & CT-TT & $414(82.6 \%)$ & $416(83.2 \%)$ & 1.00 & \\
\hline & Log-additive & - & - & - & $1.05(0.88-1.26)$ & 0.575 \\
\hline \multirow[t]{10}{*}{ rs7920624 } & \multirow[t]{2}{*}{ Allele } & A & 485 (48.79\%) & 498 (49.80\%) & $0.96(0.81-1.15)$ & 0.653 \\
\hline & & $\mathrm{T}$ & 509 (51.21\%) & $502(50.20 \%)$ & 1.00 & \\
\hline & \multirow[t]{3}{*}{ Genotype } & AA & $110(22.1 \%)$ & $123(24.6 \%)$ & $0.91(0.64-1.31)$ & 0.623 \\
\hline & & AT & $265(53.3 \%)$ & $252(50.4 \%)$ & $1.08(0.80-1.46)$ & 0.629 \\
\hline & & $\mathrm{TT}$ & $122(24.6 \%)$ & $125(25 \%)$ & 1.00 & \\
\hline & \multirow[t]{2}{*}{ Dominant } & AA-AT & 375 (75.5\%) & $375(75 \%)$ & $1.02(0.77-1.37)$ & 0.872 \\
\hline & & $\mathrm{TT}$ & $122(24.6 \%)$ & $125(25 \%)$ & 1.00 & \\
\hline & \multirow[t]{2}{*}{ Recessive } & AA & $110(22.1 \%)$ & $123(24.6 \%)$ & $0.87(0.65-1.17)$ & 0.349 \\
\hline & & AT-TT & $387(77.9 \%)$ & $377(75.4 \%)$ & 1.00 & \\
\hline & Log-additive & - & - & - & $0.96(0.80-1.15)$ & 0.637 \\
\hline \multirow[t]{10}{*}{ rs2441727 } & \multirow[t]{2}{*}{ Allele } & A & 182 (18.16\%) & 202 (20.45\%) & $0.86(0.69-1.08)$ & 0.197 \\
\hline & & G & 820 (81.84\%) & 786 (79.55\%) & 1.00 & \\
\hline & \multirow[t]{3}{*}{ Genotype } & $\mathrm{AA}$ & $17(3.4 \%)$ & 17 (3.4\%) & $0.91(0.46-1.83)$ & 0.802 \\
\hline & & $A G$ & $148(29.5 \%)$ & 168 (34\%) & $0.81(0.62-1.06)$ & 0.128 \\
\hline & & GG & $336(67.1 \%)$ & $309(62.5 \%)$ & 1.00 & \\
\hline & \multirow[t]{2}{*}{ Dominant } & $A A-A G$ & $165(32.9 \%)$ & $185(37.5 \%)$ & $0.82(0.63-1.07)$ & 0.137 \\
\hline & & GG & $336(67.1 \%)$ & 309 (62.5\%) & 1.00 & \\
\hline & \multirow[t]{2}{*}{ Recessive } & AA & $17(3.4 \%)$ & 17 (3.4\%) & $0.98(0.49-1.95)$ & 0.955 \\
\hline & & AG-GG & $484(96.6 \%)$ & 477 (96.6\%) & 1.00 & \\
\hline & Log-additive & - & - & - & $0.86(0.69-1.08)$ & 0.192 \\
\hline \multirow[t]{10}{*}{ rs7914287 } & \multirow[t]{2}{*}{ Allele } & $T$ & 355 (35.50\%) & 284 (29.22\%) & $1.33(1.10-1.61)$ & $0.003^{*}$ \\
\hline & & $C$ & 645 (64.50\%) & 688 (70.78\%) & 1.00 & \\
\hline & \multirow[t]{3}{*}{ Genotype } & $\mathrm{TT}$ & $68(13.6 \%)$ & $34(7 \%)$ & $2.21(1.41-3.47)$ & $0.001 *$ \\
\hline & & $\mathrm{TC}$ & $219(43.8 \%)$ & $216(44.4 \%)$ & $1.12(0.86-1.46)$ & 0.389 \\
\hline & & $\mathrm{CC}$ & $213(42.6 \%)$ & $236(48.6 \%)$ & 1.00 & \\
\hline & \multirow[t]{2}{*}{ Dominant } & TT-TC & $287(57.4 \%)$ & $250(51.4 \%)$ & $1.27(0.99-1.63)$ & 0.062 \\
\hline & & $C C$ & $213(42.6 \%)$ & $236(48.6 \%)$ & 1.00 & \\
\hline & Recessive & $\mathrm{TT}$ & $68(13.6 \%)$ & $34(7 \%)$ & $2.09(1.36-3.22)$ & $0.001 *$ \\
\hline & & $\mathrm{TC}-\mathrm{CC}$ & $432(86.4 \%)$ & 452 (93\%) & 1.00 & \\
\hline & Log-additive & - & - & - & $1.34(1.10-1.62)$ & $0.003^{*}$ \\
\hline
\end{tabular}

SNP, Single nucleotide polymorphisms; OR, odds ratio; Cl, Confidence interval $p<0.05$, bold text and ${ }^{\prime * \prime}$ indicate statistical significance

"-" indicates Log-additive model

$(p=0.013)$. The level of aspartate aminotransferase $(p=0.037)$, alanine aminotransferase $(p=0.044)$ and gamma-glutamyltransferase $(p=0.029)$ also had significant differences under different genotypes of rs7914287.
There was no significant difference between the remaining candidate SNPs and the level of clinical indicators (Additional file 1: Table S1). 
Table 4 The SNPS of CTNNA3 associated with susceptibility of type 2 diabetes in the subgroup tests (age and gender)

\begin{tabular}{|c|c|c|c|c|c|c|c|c|c|c|}
\hline \multirow[t]{3}{*}{ SNP ID } & \multirow[t]{3}{*}{ Model } & \multirow[t]{3}{*}{ Genotype } & \multicolumn{4}{|l|}{ Age, years } & \multicolumn{4}{|l|}{ Gender } \\
\hline & & & OR $(95 \% \mathrm{Cl})$ & $p$ & OR $(95 \% \mathrm{Cl})$ & $P$ & OR $(95 \% \mathrm{Cl})$ & $p$ & OR $(95 \% \mathrm{Cl})$ & $p$ \\
\hline & & & $\leq 60(\mathrm{~N}=500)$ & & $>60(\mathrm{~N}=502)$ & & Female $(\mathrm{N}=\mathbf{2 8 4}$ & & Male $(\mathrm{N}=718)$ & \\
\hline \multirow[t]{10}{*}{ rs10822745 } & Allele & C & $1.03(0.80-1.32)$ & 0.844 & $1.07(0.83-1.38)$ & 0.587 & $1.09(0.78-1.53)$ & 0.600 & $1.04(0.84-1.28)$ & 0.749 \\
\hline & & $\mathrm{T}$ & 1.00 & & 1.00 & & 1.00 & & 1.00 & \\
\hline & Genotype & CC & $1.09(0.65-1.85)$ & 0.739 & $1.05(0.61-1.79)$ & 0.872 & $1.20(0.61-2.36)$ & 0.601 & $1.05(0.68-1.64)$ & 0.816 \\
\hline & & CT & $0.91(0.61-1.36)$ & 0.660 & $1.21(0.81-1.81)$ & 0.348 & $1.08(0.64-1.82)$ & 0.775 & $1.09(0.78-1.52)$ & 0.613 \\
\hline & & $\mathrm{TT}$ & 1.00 & & 1.00 & & 1.00 & & 1.00 & \\
\hline & Dominant & $\mathrm{CC}-\mathrm{CT}$ & $0.96(0.65-1.40)$ & 0.819 & $1.17(0.8-1.71)$ & 0.426 & $1.11(0.68-1.82)$ & 0.675 & $1.08(0.79-1.48)$ & 0.630 \\
\hline & & $\mathrm{TT}$ & 1.00 & & 1.00 & & 1.00 & & 1.00 & \\
\hline & Recessive & CC & $1.16(0.73-1.84)$ & 0.541 & $0.93(0.58-1.51)$ & 0.771 & $1.15(0.62-2.11)$ & 0.659 & $1.00(0.68-1.48)$ & 0.999 \\
\hline & & CT-TT & 1.00 & & 1.00 & & 1.00 & & 1.00 & \\
\hline & Log-additive & - & $1.02(0.79-1.32)$ & 0.854 & $1.05(0.81-1.37)$ & 0.699 & $1.09(0.78-1.52)$ & 0.601 & $1.04(0.84-1.29)$ & 0.742 \\
\hline \multirow[t]{10}{*}{ rs7920624 } & Allele & A & $1.13(0.88-1.45)$ & 0.329 & $1.05(0.82-1.35)$ & 0.700 & $0.95(0.68-1.31)$ & 0.737 & $0.97(0.79-1.19)$ & 0.749 \\
\hline & & $\mathrm{T}$ & 1.00 & & 1.00 & & 1.00 & & 1.00 & \\
\hline & Genotype & AA & $1.28(0.76-2.14)$ & 0.350 & $1.09(0.65-1.83)$ & 0.745 & $0.89(0.46-1.73)$ & 0.727 & $0.93(0.60-1.42)$ & 0.720 \\
\hline & & AT & $1.02(0.65-1.59)$ & 0.949 & $1.47(0.94-2.29)$ & 0.092 & $1.00(0.57-1.76)$ & 1.000 & $1.11(0.78-1.59)$ & 0.567 \\
\hline & & $\mathrm{TT}$ & 1.00 & & 1.00 & & 1.00 & & 1.00 & \\
\hline & Dominant & AA-AT & $1.09(0.72-1.67)$ & 0.679 & $1.33(0.87-2.03)$ & 0.183 & $0.96(0.57-1.64)$ & 0.890 & $1.05(0.75-1.48)$ & 0.779 \\
\hline & & TT & 1.00 & & 1.00 & & 1.00 & & 1.00 & \\
\hline & Recessive & $\mathrm{AA}$ & $1.27(0.84-1.90)$ & 0.259 & $0.84(0.55-1.28)$ & 0.411 & $0.89(0.51-1.54)$ & 0.673 & $0.86(0.61-1.22)$ & 0.399 \\
\hline & & AT-TT & 1.00 & & 1.00 & & 1.00 & & 1.00 & \\
\hline & Log-additive & - & $1.14(0.88-1.47)$ & 0.336 & $1.04(0.81-1.35)$ & 0.755 & $0.94(0.68-1.32)$ & 0.733 & $0.96(0.78-1.19)$ & 0.733 \\
\hline \multirow[t]{10}{*}{ rs2441727 } & Allele & A & $0.98(0.71-1.35)$ & 0.887 & $0.78(0.58-1.07)$ & 0.122 & $0.89(0.60-1.33)$ & 0.581 & $0.85(0.65-1.11)$ & 0.238 \\
\hline & & G & 1.00 & & 1.00 & & 1.00 & & 1.00 & \\
\hline & Genotype & $\mathrm{AA}$ & $0.46(0.11-1.88)$ & 0.281 & $0.73(0.49-1.67)$ & 0.748 & $0.95(0.36-2.03)$ & 0.933 & $0.82(0.33-2.06)$ & 0.677 \\
\hline & & $A G$ & $0.86(0.43-1.26)$ & 0.757 & $0.58(0.39-0.87)$ & $0.008^{*}$ & $0.80(0.48-1.31)$ & 0.371 & $0.82(0.59-1.13)$ & 0.218 \\
\hline & & GG & 1.00 & & 1.00 & & 1.00 & & 1.00 & \\
\hline & Dominant & $A A-A G$ & $0.84(0.70-1.48)$ & 0.931 & $0.64(0.44-0.93)$ & $0.021^{*}$ & $0.83(0.51-1.33)$ & 0.433 & $0.82(0.60-1.12)$ & 0.206 \\
\hline & & GG & 1.00 & & 1.00 & & 1.00 & & 1.00 & \\
\hline & Recessive & AA & $0.45(0.11-1.84)$ & 0.267 & $0.57(0.60-0.76)$ & 0.455 & $0.94(0.40-1.23)$ & 0.812 & $0.88(0.35-2.19)$ & 0.775 \\
\hline & & AG-GG & 1.00 & & 1.00 & & 1.00 & & 1.00 & \\
\hline & Log-additive & - & $0.96(0.68-1.36)$ & 0.830 & $0.77(0.57-1.05)$ & 0.103 & $0.89(0.60-1.33)$ & 0.579 & $0.84(0.64-1.11)$ & 0.228 \\
\hline \multirow[t]{10}{*}{ rs7914287 } & Allele & $\mathrm{T}$ & $1.31(1.00-1.72)$ & 0.052 & $1.37(1.06-1.79)$ & $0.018^{*}$ & $1.34(0.94-1.90)$ & 0.107 & $2.33(1.07-3.67)$ & $0.012^{*}$ \\
\hline & & C & 1.00 & & 1.00 & & 1.00 & & 1.00 & \\
\hline & Genotype & $\mathrm{TT}$ & $2.71(1.36-5.42)$ & 0.057 & $2.22(1.18-4.17)$ & $0.013^{*}$ & $2.26(0.97-5.22)$ & 0.058 & $2.20(1.29-3.77)$ & $0.004^{*}$ \\
\hline & & $\mathrm{TC}$ & $0.99(0.68-1.44)$ & 0.952 & $1.43(0.97-2.11)$ & 0.070 & $1.11(0.67-1.83)$ & 0.681 & $1.12(0.82-1.54)$ & 0.461 \\
\hline & & CC & 1.00 & & 1.00 & & 1.00 & & 1.00 & \\
\hline & Dominant & TT-TC & $1.18(0.82-1.68)$ & 0.372 & $1.56(1.08-2.25)$ & $0.019^{*}$ & $1.27(0.79-2.04)$ & 0.330 & $1.27(0.95-1.71)$ & 0.113 \\
\hline & & CC & 1.00 & & 1.00 & & 1.00 & & 1.00 & \\
\hline & Recessive & $\mathrm{TT}$ & $2.73(1.40-5.32)$ & 0.120 & $1.85(1.02-3.35)$ & $0.044^{*}$ & $2.14(0.96-4.76)$ & 0.063 & $2.08(1.24-3.49)$ & $0.005^{*}$ \\
\hline & & TC-CC & 1.00 & & 1.00 & & 1.00 & & 1.00 & \\
\hline & Log-additive & - & $1.33(1.01-1.75)$ & 0.064 & $1.47(1.11-1.94)$ & $0.007^{*}$ & $1.35(0.94-1.93)$ & 0.106 & $2.33(1.06-3.67)$ & $0.013^{*}$ \\
\hline
\end{tabular}

SNP, Single nucleotide polymorphisms; OR, odds ratio; $\mathrm{Cl}$, Confidence interval $p<0.05$, bold text and '*'indicate statistical significance

"-" indicates Log-additive model 
Table 5 The SNPS of CTNNA3 associated with susceptibility of type 2 diabetes in the subgroup tests (smoking and drinking)

\begin{tabular}{|c|c|c|c|c|c|c|c|c|c|c|}
\hline \multirow[t]{3}{*}{ SNP ID } & \multirow[t]{3}{*}{ Model } & \multirow[t]{3}{*}{ Genotype } & \multicolumn{4}{|l|}{ Smoking } & \multicolumn{4}{|l|}{ Drinking } \\
\hline & & & OR $(95 \% \mathrm{Cl})$ & $p$ & OR $(95 \% \mathrm{Cl})$ & $p$ & OR $(95 \% \mathrm{Cl})$ & $p$ & OR $(95 \% \mathrm{Cl})$ & $p$ \\
\hline & & & No $(\mathrm{N}=454)$ & & Yes (N=342) & & No (N=528) & & Yes $(\mathrm{N}=236)$ & \\
\hline \multirow[t]{10}{*}{ rs10822745 } & Allele & C & $0.96(0.70-1.31)$ & 0.777 & $1.11(0.85-1.46)$ & 0.447 & $1.13(0.78-1.63)$ & 0.524 & $1.05(0.80-1.39)$ & 0.715 \\
\hline & & $\mathrm{T}$ & 1.00 & & 1.00 & & 1.00 & & 1.00 & \\
\hline & Genotype & CC & $0.79(0.40-1.56)$ & 0.500 & $1.26(0.73-2.17)$ & 0.407 & $1.26(0.57-2.76)$ & 0.568 & $0.98(0.57-1.69)$ & 0.954 \\
\hline & & $\mathrm{CT}$ & $1.25(0.76-2.05)$ & 0.377 & $1.11(0.72-1.71)$ & 0.628 & $1.21(0.68-2.14)$ & 0.518 & $1.43(0.93-2.21)$ & 0.107 \\
\hline & & $\mathrm{TT}$ & 1.00 & & 1.00 & & 1.00 & & 1.00 & \\
\hline & Dominant & CC-CT & $1.13(0.70-1.8)$ & 0.619 & $1.15(0.77-1.73)$ & 0.488 & $1.22(0.71-2.11)$ & 0.476 & $1.28(0.86-1.91)$ & 0.230 \\
\hline & & $\mathrm{TT}$ & 1.00 & & 1.00 & & 1.00 & & 1.00 & \\
\hline & Recessive & CC & $0.69(0.38-1.27)$ & 0.233 & $1.18(0.73-1.91)$ & 0.496 & $1.12(0.55-2.27)$ & 0.750 & $0.80(0.50-1.30)$ & 0.375 \\
\hline & & CT-TT & 1.00 & & 1.00 & & 1.00 & & 1.00 & \\
\hline & Log-additive & - & $0.95(0.68-1.33)$ & 0.773 & $1.12(0.86-1.46)$ & 0.403 & $1.14(0.78-1.66)$ & 0.505 & $1.05(0.79-1.37)$ & 0.753 \\
\hline \multirow[t]{10}{*}{ rs7920624 } & Allele & A & $1.01(0.74-1.39)$ & 0.931 & $0.95(0.73-1.25)$ & 0.733 & $1.10(0.77-1.59)$ & 0.598 & $0.99(0.75-1.29)$ & 0.921 \\
\hline & & $\mathrm{T}$ & 1.00 & & 1.00 & & 1.00 & & 1.00 & \\
\hline & Genotype & AA & $1.03(0.54-1.97)$ & 0.918 & $0.89(0.52-1.51)$ & 0.659 & $1.23(0.60-2.53)$ & 0.574 & $0.97(0.56-1.65)$ & 0.899 \\
\hline & & AT & $1.52(0.88-2.63)$ & 0.131 & $1.02(0.65-1.60)$ & 0.943 & $1.36(0.73-2.55)$ & 0.334 & $1.37(0.86-2.19)$ & 0.182 \\
\hline & & $\mathrm{TT}$ & 1.00 & & 1.00 & & 1.00 & & 1.00 & \\
\hline & Dominant & AA-AT & $1.36(0.81-2.27)$ & 0.249 & $0.97(0.63-1.49)$ & 0.898 & $1.31(0.73-2.36)$ & 0.361 & $1.22(0.79-1.89)$ & 0.363 \\
\hline & & TT & 1.00 & & 1.00 & & 1.00 & & 1.00 & \\
\hline & Recessive & $\mathrm{AA}$ & $0.77(0.46-1.31)$ & 0.342 & $0.88(0.56-1.37)$ & 0.567 & $1.01(0.56-1.83)$ & 0.978 & $0.78(0.50-1.23)$ & 0.286 \\
\hline & & AT-TT & 1.00 & & 1.00 & & 1.00 & & 1.00 & \\
\hline & Log-additive & - & $1.02(0.74-1.42)$ & 0.892 & $0.94(0.72-1.23)$ & 0.674 & $1.11(0.78-1.59)$ & 0.563 & $0.99(0.75-1.31)$ & 0.937 \\
\hline \multirow[t]{10}{*}{ rs2441727 } & Allele & A & $1.01(0.67-1.53)$ & 0.953 & $0.79(0.56-1.10)$ & 0.154 & $0.97(0.62-1.51)$ & 0.896 & $0.80(0.57-1.12)$ & 0.191 \\
\hline & & G & 1.00 & & 1.00 & & 1.00 & & 1.00 & \\
\hline & Genotype & $\mathrm{AA}$ & $3.08(0.36-6.41)$ & 0.305 & $1.32(0.41-4.29)$ & 0.645 & $0.67(0.15-2.95)$ & 0.599 & $2.60(0.57-5.82)$ & 0.215 \\
\hline & & $A G$ & $0.88(0.55-1.42)$ & 0.607 & $0.61(0.41-0.92)$ & $0.018^{*}$ & $1.08(0.63-1.87)$ & 0.778 & $0.63(0.42-0.94)$ & $0.025 *$ \\
\hline & & GG & 1.00 & & 1.00 & & 1.00 & & 1.00 & \\
\hline & Dominant & AA-AG & $0.94(0.58-1.50)$ & 0.780 & $0.65(0.44-0.97)$ & $0.034^{*}$ & $1.04(0.61-1.76)$ & 0.892 & $0.69(0.46-1.03)$ & 0.071 \\
\hline & & GG & 1.00 & & 1.00 & & 1.00 & & 1.00 & \\
\hline & Recessive & $\mathrm{AA}$ & $0.82(0.38-1.34)$ & 0.287 & $0.57(0.49-1.06)$ & 0.448 & $0.65(0.15-2.83)$ & 0.570 & $0.27(0.68-1.83)$ & 0.144 \\
\hline & & $A G-G G$ & 1.00 & & 1.00 & & 1.00 & & 1.00 & \\
\hline & Log-additive & - & $0.93(0.66-1.55)$ & 0.963 & $0.75(0.54-1.06)$ & 0.108 & $0.98(0.62-1.56)$ & 0.947 & $0.83(0.58-1.17)$ & 0.281 \\
\hline \multirow[t]{10}{*}{ rs7914287 } & Allele & $\mathrm{T}$ & $1.36(0.97-1.91)$ & 0.078 & $2.36(1.01-4.82)$ & $0.023^{*}$ & $1.20(0.81-1.77)$ & 0.368 & $1.41(1.04-2.91)$ & $0.025^{*}$ \\
\hline & & C & 1.00 & & 1.00 & & 1.00 & & 1.00 & \\
\hline & Genotype & $\mathrm{TT}$ & $1.91(0.87-4.21)$ & 0.109 & $2.50(1.18-5.32)$ & $0.017^{*}$ & $1.33(0.55-3.20)$ & 0.527 & $2.11(1.34-5.20)$ & $0.008^{*}$ \\
\hline & & $\mathrm{TC}$ & $1.29(0.80-2.07)$ & 0.302 & $1.06(0.71-1.58)$ & 0.788 & $1.21(0.70-2.09)$ & 0.501 & $1.08(0.72-1.62)$ & 0.721 \\
\hline & & CC & 1.00 & & 1.00 & & 1.00 & & 1.00 & \\
\hline & Dominant & TT-TC & $1.39(0.89-2.19)$ & 0.152 & $1.23(0.83-1.81)$ & 0.299 & $1.23(0.73-2.07)$ & 0.436 & $1.28(0.86-1.90)$ & 0.219 \\
\hline & & CC & 1.00 & & 1.00 & & 1.00 & & 1.00 & \\
\hline & Recessive & $\mathrm{TT}$ & $1.69(0.79-3.60)$ & 0.175 & $2.44(1.18-5.05)$ & $0.016^{*}$ & $1.21(0.52-2.79)$ & 0.655 & $2.00(1.33-4.77)$ & $0.008^{*}$ \\
\hline & & $\mathrm{TC}-\mathrm{CC}$ & 1.00 & & 1.00 & & 1.00 & & 1.00 & \\
\hline & Log-additive & - & $1.35(0.96-1.89)$ & 0.089 & $1.34(0.99-1.80)$ & 0.055 & $1.17(0.79-1.73)$ & 0.428 & $1.41(1.04-1.91)$ & $0.027^{*}$ \\
\hline
\end{tabular}

$\mathrm{OR}$, odds ratio; $\mathrm{Cl}$, confidence interval

$p<0.05$, bold text and '*' indicate statistical significance

"-" indicates Log-additive model 
Table 6 The SNPS of CTNNA3 associated with susceptibility of type 2 diabetes in the subgroup tests (BMI)

\begin{tabular}{|c|c|c|c|c|c|c|}
\hline \multirow[t]{3}{*}{ SNP ID } & \multirow[t]{3}{*}{ Model } & \multirow[t]{3}{*}{ Genotype } & \multicolumn{4}{|l|}{ BMI } \\
\hline & & & OR $(95 \% \mathrm{Cl})$ & $p$ & OR $(95 \% \mathrm{Cl})$ & $p$ \\
\hline & & & $\leq 24(\mathrm{~N}=326)$ & & $>24(\mathrm{~N}=426)$ & \\
\hline \multirow[t]{6}{*}{ rs10822745 } & Allele & $C / T$ & $1.12(0.81-1.54)$ & 0.499 & $0.89(0.68-1.17)$ & 0.415 \\
\hline & Homozygote & $\mathrm{CC} / \mathrm{TT}$ & $1.43(0.69-2.95)$ & 0.332 & $0.77(0.44-1.37)$ & 0.375 \\
\hline & Heterozygote & $\mathrm{CT}$ & $0.95(0.58-1.56)$ & 0.839 & $1.06(0.68-1.67)$ & 0.793 \\
\hline & Dominant & CC-CT/TT & $1.04(0.64-1.67)$ & 0.881 & $0.97(0.63-1.50)$ & 0.905 \\
\hline & Recessive & CC/CT-TT & $1.48(0.77-2.85)$ & 0.244 & $0.74(0.45-1.22)$ & 0.237 \\
\hline & Log-additive & - & $1.13(0.81-1.59)$ & 0.465 & $0.90(0.68-1.19)$ & 0.448 \\
\hline \multirow[t]{6}{*}{ rs7920624 } & Allele & $\mathrm{A} / \mathrm{T}$ & $1.05(0.76-1.44)$ & 0.763 & $1.12(0.85-1.47)$ & 0.430 \\
\hline & Homozygote & $\mathrm{AA} / \mathrm{TT}$ & $1.13(0.56-2.26)$ & 0.733 & $1.25(0.72-2.17)$ & 0.422 \\
\hline & Heterozygote & AT & $1.16(0.66-2.04)$ & 0.610 & $1.28(0.81-2.02)$ & 0.294 \\
\hline & Dominant & AA-AT/TT & $1.15(0.67-1.99)$ & 0.613 & $1.27(0.83-1.95)$ & 0.276 \\
\hline & Recessive & AA/AT-TT & $1.01(0.58-1.77)$ & 0.963 & $1.07(0.67-1.72)$ & 0.765 \\
\hline & Log-additive & - & $1.06(0.75-1.51)$ & 0.727 & $1.13(0.86-1.49)$ & 0.385 \\
\hline \multirow[t]{6}{*}{ rs2441727 } & Allele & $A / G$ & $0.72(0.48-1.08)$ & 0.115 & $1.00(0.70-1.41)$ & 0.993 \\
\hline & Homozygote & $\mathrm{AA} / \mathrm{GG}$ & $1.10(0.19-6.24)$ & 0.913 & $1.32(0.32-5.51)$ & 0.705 \\
\hline & Heterozygote & $\mathrm{AG} / \mathrm{GG}$ & $0.64(0.39-1.03)$ & 0.068 & $0.96(0.63-1.45)$ & 0.829 \\
\hline & Dominant & $\mathrm{AA}-\mathrm{AG} / \mathrm{GG}$ & $0.66(0.41-1.06)$ & 0.082 & $0.97(0.65-1.46)$ & 0.896 \\
\hline & Recessive & $\mathrm{AA} / \mathrm{AG}-\mathrm{GG}$ & $1.30(0.23-7.31)$ & 0.765 & $1.34(0.32-5.56)$ & 0.689 \\
\hline & Log-additive & - & $1.16(0.77-1.74)$ & 0.484 & $1.00(0.69-1.45)$ & 0.991 \\
\hline \multirow[t]{6}{*}{ rs7914287 } & Allele & $\mathrm{T} / \mathrm{C}$ & $1.57(1.11-2.24)$ & 0.072 & $1.45(1.08-1.96)$ & $0.014 *$ \\
\hline & Homozygote & $\mathrm{TT} / \mathrm{CC}$ & $3.66(1.43-5.36)$ & 0.120 & $2.86(1.32-6.24)$ & $0.008^{*}$ \\
\hline & Heterozygote & $\mathrm{TC} / \mathrm{CC}$ & $1.27(0.79-2.06)$ & 0.325 & $1.24(0.82-1.88)$ & 0.302 \\
\hline & Dominant & TT-TC/CC & $1.52(0.96-2.42)$ & 0.073 & $1.42(0.96-2.11)$ & 0.080 \\
\hline & Recessive & TT/TC-CC & $2.25(1.31-4.07)$ & 0.093 & $2.58(1.22-4.47)$ & $0.014 *$ \\
\hline & Log-additive & - & $1.60(1.12-2.29)$ & 0.110 & $1.48(1.09-2.01)$ & $0.013^{*}$ \\
\hline
\end{tabular}

$\mathrm{OR}$, odds ratio; $\mathrm{Cl}$, confidence interval

$p<0.05$, bold text and '*'indicate statistical significance

"-" indicates Log-additive model

\section{FPRP analysis}

The results of FPRP analysis showed that (Additional file 2: Table S2) the association between CTNNA3 rs7914287 and T2D risk in drinking participants was not noteworthy at the prior probability level of 0.25 and FPRP threshold of 0.2 . The FPRP of the remaining significant results were all less than 0.2 , which means that these positive results were noteworthy.

\section{LD and haplotype analysis}

The results of linkage disequilibrium and haplotype analysis of CTNNA3 polymorphism showed (Fig. 1): there is an LD block ( $\left.D^{\prime}=0.968, R^{2}=0.665\right)$ composed of 2 SNPs (rs10822745 and rs7920624). However, logistic regression results showed that there was no statistically significant difference among the CTNNA3 haplotype frequencies in the cases and controls (Additional file 3: Table S3).

\section{Analysis of MDR}

We used MDR to analyze and predict the interaction between SNP-SNP. Figure 2 was dendrogram analysis of SNP-SNP interaction. The color in the figure represents whether the effect of SNP-SNP on T2D risk is synergistic or redundant. The color in the figure represents whether the effect of SNP-SNP on T2D risk is synergistic or redundant. The blue line in the dendrogram indicates that candidate SNPs have a redundant role in regulating T2D risk (Fig. 2). The results (Table 9) showed that the four loci models (rs10822745, rs7920624, rs2441727, rs7914287) have the highest test accuracy. However, considering the small sample size, the rs7920624 and rs2441727 two-site model was regard as the overall best model, with a test accuracy of 0.541 and a good CVC (9/10). 
Table 7 The SNPS of CTNNA3 associated with susceptibility of type 2 diabetes in the subgroup tests (no retinal degeneration and course of type 2 diabetes)

\begin{tabular}{|c|c|c|c|c|c|c|}
\hline \multirow[t]{2}{*}{ SNP ID } & \multirow[t]{2}{*}{ Model } & \multirow[t]{2}{*}{ Genotype } & \multicolumn{2}{|c|}{$\begin{array}{l}\text { No retinal degeneration } \\
\text { (No retinal degeneration in cases } \\
\text { vs. } \leq \text { control) } \\
(\mathrm{N}=741)\end{array}$} & \multicolumn{2}{|c|}{$\begin{array}{l}\text { Course of type } 2 \text { diabetes }(>10 \\
\text { vs. } \leq 10 \text { years }) \\
(N=500)\end{array}$} \\
\hline & & & OR $(95 \% \mathrm{Cl})$ & $p$ & OR $(95 \% \mathrm{Cl})$ & $p$ \\
\hline \multirow[t]{6}{*}{ rs10822745 } & Allele & $\mathrm{C} / \mathrm{T}$ & $1.07(0.86-1.33)$ & 0.552 & $0.74(0.57-0.96)$ & $0.022^{*}$ \\
\hline & Homozygote & $\mathrm{CC} / \mathrm{TT}$ & $1.07(0.66-1.72)$ & 0.795 & $0.49(0.28-0.88)$ & $0.017^{*}$ \\
\hline & Heterozygote & $\mathrm{CT}$ & $1.30(0.92-1.85)$ & 0.139 & $0.83(0.55-1.25)$ & 0.368 \\
\hline & Dominant & $\mathrm{CC}-\mathrm{CT} / \mathrm{TT}$ & $1.24(0.89-1.74)$ & 0.204 & $0.73(0.49-1.09)$ & 0.120 \\
\hline & Recessive & CC/CT-TT & $0.90(0.59-1.38)$ & 0.631 & $0.55(0.33-0.94)$ & $0.027^{*}$ \\
\hline & Log-additive & - & $1.07(0.86-1.35)$ & 0.540 & $0.73(0.55-0.96)$ & $0.023^{*}$ \\
\hline \multirow[t]{6}{*}{ rs7920624 } & Allele & $\mathrm{A} / \mathrm{T}$ & $0.95(0.76-1.18)$ & 0.647 & $1.33(1.03-1.72)$ & $0.030 *$ \\
\hline & Homozygote & $\mathrm{AA} / \mathrm{TT}$ & $0.88(0.55-1.41)$ & 0.602 & $1.73(1.00-3.01)$ & 0.052 \\
\hline & Heterozygote & AT & $1.29(0.88-1.90)$ & 0.187 & $1.50(0.94-2.40)$ & 0.091 \\
\hline & Dominant & AA-AT/TT & $1.16(0.80-1.67)$ & 0.430 & $1.56(1.00-2.45)$ & 0.051 \\
\hline & Recessive & AA/AT-TT & $0.74(0.50-1.08)$ & 0.118 & $1.30(0.84-2.03)$ & 0.238 \\
\hline & Log-additive & - & $0.95(0.76-1.19)$ & 0.640 & $1.31(1.00-1.73)$ & 0.051 \\
\hline \multirow[t]{6}{*}{ rs 2441727} & Allele & $A / G$ & $0.81(0.61-1.08)$ & 0.152 & $1.33(1.03-1.72)$ & $0.030 *$ \\
\hline & Homozygote & $\mathrm{AA} / \mathrm{GG}$ & $0.56(0.20-1.54)$ & 0.261 & $0.61(0.22-1.68)$ & 0.336 \\
\hline & Heterozygote & $A G / G G$ & $0.83(0.59-1.16)$ & 0.268 & $0.49(0.32-0.76)$ & $0.001 *$ \\
\hline & Dominant & $\mathrm{AA}-\mathrm{AG} / \mathrm{GG}$ & $0.80(0.58-1.11)$ & 0.188 & $0.50(0.33-0.76)$ & $0.001 *$ \\
\hline & Recessive & AA/AG-GG & $0.59(0.22-1.64)$ & 0.314 & $0.74(0.27-2.04)$ & 0.558 \\
\hline & Log-additive & - & $0.80(0.60-1.08)$ & 0.140 & $0.59(0.41-0.84)$ & $0.003^{*}$ \\
\hline \multirow[t]{6}{*}{ rs7914287 } & Allele & $\mathrm{T} / \mathrm{C}$ & $1.31(1.04-1.66)$ & $0.022^{*}$ & $1.10(0.85-1.44)$ & 0.466 \\
\hline & Homozygote & TT/CC & $2.20(1.29-3.75)$ & $0.004^{*}$ & $1.23(0.70-2.16)$ & 0.479 \\
\hline & Heterozygote & $\mathrm{TC} / \mathrm{CC}$ & $1.07(0.77-1.49)$ & 0.682 & $0.89(0.60-1.34)$ & 0.587 \\
\hline & Dominant & TT-TC/CC & $1.23(0.90-1.68)$ & 0.200 & $0.97(0.66-1.41)$ & 0.859 \\
\hline & Recessive & $\mathrm{TT} / \mathrm{TC}-\mathrm{CC}$ & $2.13(1.28-3.54)$ & $0.003^{*}$ & $1.30(0.76-2.21)$ & 0.334 \\
\hline & Log-additive & - & $1.32(1.04-1.68)$ & $0.021^{*}$ & $1.05(0.80-1.37)$ & 0.721 \\
\hline
\end{tabular}

$\mathrm{OR}$, odds ratio; $\mathrm{Cl}$, confidence interval

$p<0.05$, bold text and ${ }^{\prime * \prime}$ indicate statistical significance

"_" indicates Log-additive model

\section{Discussion}

The incidence of Type 2 diabetes has increased significantly worldwide, and the number of T2D patients in many countries is increasing year by year [1]. More and more studies have confirmed that genetic factors play an indispensable role in the T2D risk [2]. More and more efforts are also devoted to improving the status quo of T2D, but there are still some clinical challenges to be overcome. For example, the existing clinical markers are not fully applicable to clinical diagnosis [25]. It was estimated that the genetic signals that have been discovered can only explain $2 \%$ of the T2D risk [16]. Therefore, it is still an arduous and long-term task to discover more genetic polymorphisms related to T2D risk.

There are relatively few studies on the association between CTNNA3 genetic polymorphisms and diseases. In recent reports, CTNNA3 single nucleotide polymorphisms in African populations can be used as new genetic signals for MetS, and MetS risk is closely associated with T2D risk $[19,20]$. Our study is the first to explore the association between CTNNA3 SNPs and T2D risk in Chinese Han population, and we found strong evidence of potential association between them. In general, among the 4 candidate SNPs, only CTNNA3 rs7914287 was significantly associated with T2D risk in the Chinese Han population under allele, homozygous, recessive and log-additive models. It may be a risk factor for T2D. We found no evidence that the remaining three SNPs are associated with T2D risk among participants.

Type 2 diabetes is most common in the elderly, but due to lack of physical activity and healthy eating habits [26-28], there are more and more obese patients among 
Table 8 Clinical characteristics of patients $(N=501)$ based on the genotypes of selected SNPS

\begin{tabular}{|c|c|c|c|c|c|c|c|c|}
\hline \multirow[t]{2}{*}{ Characteristics } & \multicolumn{4}{|l|}{ rs10822745 } & \multicolumn{4}{|l|}{ rs7914287 } \\
\hline & TT & TC & $\mathrm{CC}$ & $p$ & TT & TC & $\mathrm{CC}$ & $p$ \\
\hline FBS & $7.22 \pm 2.93$ & $7.46 \pm 3.46$ & $7.23 \pm 4.01$ & 0.770 & $7.34 \pm 3.03$ & $7.31 \pm 3.44$ & $7.39 \pm 3.48$ & 0.976 \\
\hline GHbAlc & $8.11 \pm 1.99$ & $8.17 \pm 2.16$ & $7.71 \pm 1.83$ & 0.212 & $7.6 \pm 1.79$ & $8.18 \pm 1.99$ & $8.11 \pm 2.2$ & 0.135 \\
\hline TC & $3.5 \pm 1.48$ & $3.57 \pm 1.48$ & $3.73 \pm 4.43$ & 0.763 & $3.32 \pm 1.59$ & $3.64 \pm 3.03$ & $3.59 \pm 1.47$ & 0.598 \\
\hline TG & $2.89 \pm 3.41$ & $2.66 \pm 2.68$ & $2.57 \pm 2.41$ & 0.670 & $2.44 \pm 3.55$ & $2.83 \pm 2.6$ & $2.69 \pm 2.97$ & 0.651 \\
\hline $\mathrm{HDL}$ & $1.03 \pm 0.28$ & $0.99 \pm 0.24$ & $1.26 \pm 1.63$ & $0.013^{*}$ & $1.08 \pm 0.33$ & $1.1 \pm 1.04$ & $0.99 \pm 0.25$ & 0.306 \\
\hline Urea & $6.36 \pm 2.39$ & $6.76 \pm 3.94$ & $6.1 \pm 2.19$ & 0.206 & $6.27 \pm 1.71$ & $6.4 \pm 2.44$ & $6.72 \pm 4.21$ & 0.494 \\
\hline $\mathrm{Cr}$ & $70.78 \pm 55.46$ & $73.38 \pm 56.92$ & $65.54 \pm 29.61$ & 0.493 & $68.02 \pm 29.28$ & $68.17 \pm 26.33$ & $75.44 \pm 74.01$ & 0.320 \\
\hline Cys-c & $0.99 \pm 0.47$ & $1.02 \pm 0.48$ & $1 \pm 0.34$ & 0.709 & $0.98 \pm 0.38$ & $1.03 \pm 0.34$ & $1 \pm 0.57$ & 0.690 \\
\hline AST & $21.57 \pm 13.08$ & $22.01 \pm 16.46$ & $21.49 \pm 11.82$ & 0.939 & $22.62 \pm 12.84$ & $23.41 \pm 19.17$ & $19.84 \pm 8.59$ & $0.037^{*}$ \\
\hline ALT & $24.68 \pm 24.87$ & $24.62 \pm 27.39$ & $25.22 \pm 23.21$ & 0.982 & $23.08 \pm 14.14$ & $28.07 \pm 34.91$ & $21.97 \pm 15.68$ & $0.044^{*}$ \\
\hline GGT & $30.88 \pm 24.8$ & $34.61 \pm 39.41$ & $32.67 \pm 57.54$ & 0.652 & $29.63 \pm 19.1$ & $38.54 \pm 54.75$ & $28.72 \pm 20.7$ & $0.029^{*}$ \\
\hline $\mathrm{LPa}$ & $205.49 \pm 210.61$ & $214.26 \pm 223.75$ & $246.77 \pm 241.11$ & 0.392 & $199.82 \pm 199.91$ & $230.56 \pm 219.36$ & $209.44 \pm 233.49$ & 0.516 \\
\hline
\end{tabular}

$p<0.05$, bold text and ${ }^{\prime \prime \prime}$ represent statistical significance

FBS, fasting blood glucose; GHbA1c, glycosylated hemoglobin A1c; TC, total cholesterol; TG, triacylglycerol; HDL, high density lipoprotein; Cr, creatinine; Cys-c, cystatin c; AST, aspartate aminotransferase; ALT, alanine aminotransferase; GGT, gamma-glutamyltransferase; LPa, lysophosphatidic acid

children, adolescents and young people, which in turn leads to type 2 diabetes [29]. Therefore, we divide the participants according to the current status of T2D incidence and the potential risk factors of T2D for subgroup analysis, with a view to provide a valuable reference for T2D risk assessment in specific populations. And

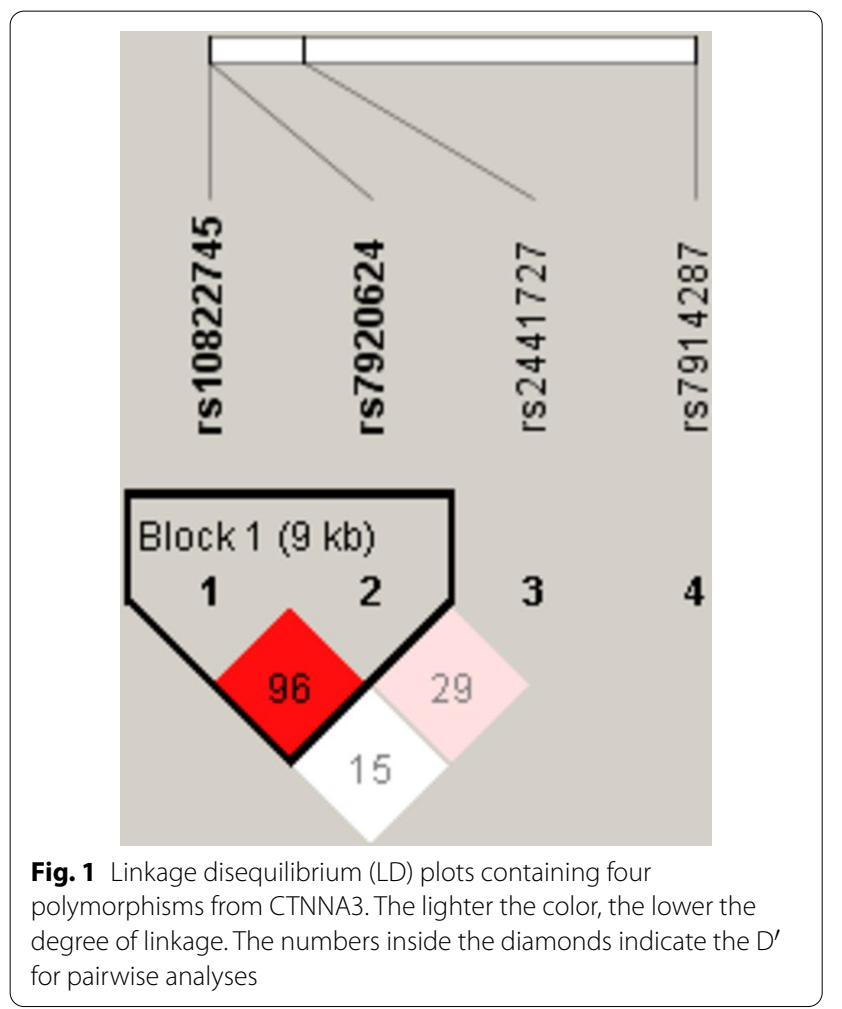

subgroup analysis for potential risk factors is an effective way to remove the influence of confounding factors. Alcohol [21], smoking [22], and aging [23] have been reported as risk factors for T2D. In this study, rs2441727 significantly reduced the T2D risk among participants who were $>60$ years old, smoking, or drinking, and it also showed a trend to reduce the risk of T2D among participants who were $\leq 60$ years old, no smoking/drinking. Our results indicate that CTNNA3-rs2441727 may be a protective factor for T2D in Chinese Han population, and this protective effect is not affected by the potential environmental risk factors of T2D. However, a large sample size and further verification tests are necessary to ensure that our results are more accurate.

Nevertheless, our study is the first to report the correlation between CTNNA3-rs2441727 and T2D risk.

Whether in the overall or subgroup analysis (age $>60$ years old, smoking, drinking, male, BMI $>24$, and no retinal degeneration), CTNNA3-rs7914287 can increase the T2D risk under multiple genetic models among participants. However, it is worth noting that in the overall analysis, under the allelic inheritance model, the allele ' $\mathrm{T}$ ' of rs7914287 seemed to show a tendency to reduce the risk of T2D, the result was not significant. We speculated that potential risk factors such as age $>60$ years old, males, smoking or drinking may promote the allele ' $\mathrm{T}$ ' of rs7914287 to become a risk factor for T2D. In addition, we also found that the results of our study are similar to previous studies: KautzkyWiller et al. have reported that T2D risk has gender differences. And this gender difference may be affected by environmental factors such as age and obesity rate 


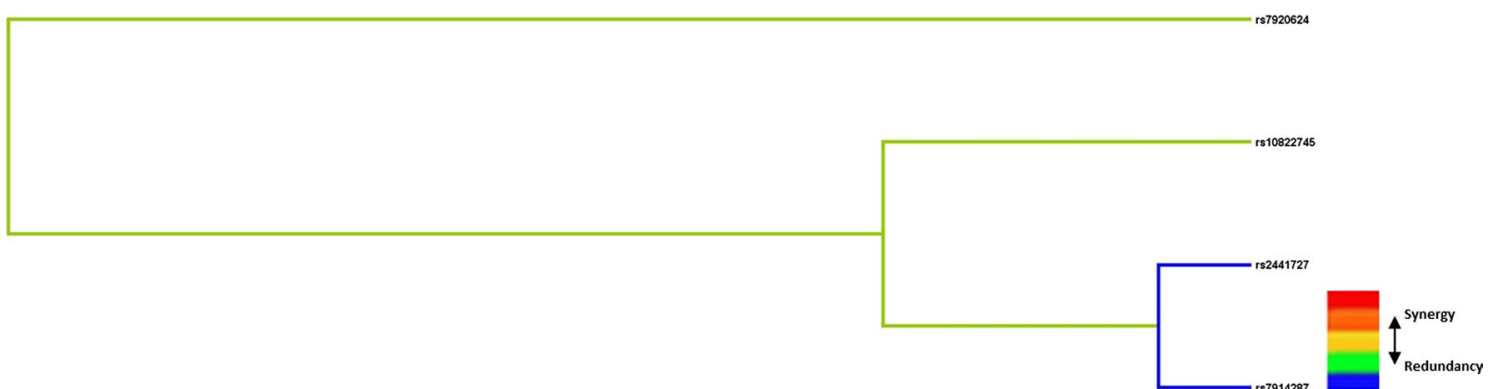

Fig. 2 Dendrogram analysis of SNP-SNP interaction. The colors in the tree diagram represent synergy or redundancy

Table 9 SNP-SNP interaction models analyzed by the MDR method

\begin{tabular}{|c|c|c|c|c|c|}
\hline Model & Training Bal. Acc & Testing Bal. Acc & OR $(95 \% \mathrm{CI})$ & $p$ value & CVC \\
\hline rs7914287 & 0.537 & 0.520 & $1.35(1.05-1.73)$ & 0.0191 & $9 / 10$ \\
\hline rs7920624, rs2441727 & 0.557 & 0.541 & $1.58(1.23-2.04)$ & 0.0003 & $9 / 10$ \\
\hline rs7920624, rs2441727, rs7914287 & 0.582 & 0.525 & $1.96(1.52-2.54)$ & $<0.0001$ & $9 / 10$ \\
\hline rs10822745, rs7920624, rs2441727, rs7914287 & 0.607 & 0.543 & $2.41(1.86-3.12)$ & $<0.0001$ & $10 / 10$ \\
\hline
\end{tabular}

MDR, multifactor dimensionality reduction; Bal. Acc., balanced accuracy; CVC, cross-validation consistency; OR, odds ratio; $95 \% \mathrm{Cl}$, $95 \%$ confidence interval $p$ values were calculated using $\mathrm{x}^{2}$ tests; $p<0.05$ and bold text indicate statistical significance

[30]. Our study also found that the association between CTNNA3-rs7914287 and T2D risk had gender differences. Increased BMI is strongly associated with T2D risk [31]. Our study also found that rs7914287 was significantly associated with T2D risk among participants with BMI $>24$. In addition to the above findings, we found that CTNNA3-rs7914287 was a risk factor for T2D patients with no retinal degeneration. However, numerous studies have reported that retinal degeneration is closely related to T2D [32, 33]. Combined with the results of this study, CTNNA3-rs7914287 can significantly increase the risk of T2D participants and may not be affected by retinal degeneration or not.

In summary, rs7914287 is a risk factor for T2D. And potential risk factors such as age $>60$ years old, males, smoking or drinking et al. may have a synergistic effect with rs7914287 in increasing the risk of T2D.

And we were also pleasantly surprised to find that the levels of AST, ALT and GGT of T2D patients were significantly different under different genotypes of rs7914287. And there were studies have reported that the increase in AST, ALT and GGT levels may be related to the increased risk of T2D [32, 34, 35]. In our study, the T2D participants had significantly higher AST, ALT and GGT levels under the rs7914287 TC genotype. Therefore, we speculate that CTNNA3-rs7914287 may increase the T2D risk by affecting the levels of AST, ALT and GGT. But this is just a speculation, CTNNA3-rs7914287 mechanism in the pathogenesis of T2D risk remains unclear, further research is needed. Nevertheless, our study suggest that CTNNA3 genetic polymorphism may be a new genetic signal of T2D risk in Chinese Han population, providing new ideas and valuable references for clinical early prevention and individualized treatment of T2D in Chinese Han population.

It is worth noting that our study still has certain limitations. If the sample size is further expanded for research verification, it will be more helpful to confirm the results of our study.

\section{Conclusion}

In summary, we found that CTNNA3 genetic polymorphisms can be used as a new genetic signal of T2D risk in Chinese Han population. Especially, CTNNA3-rs7914287 showed an outstanding and significant association with T2D risk in both overall analysis and subgroup analysis. Our study has provided valuable data supplements for the T2D susceptibility loci in Chinese Han population.

\section{Supplementary Information}

The online version contains supplementary material available at https://doi. org/10.1186/s12920-021-01105-8.

Additional file 1. Supplemental table 1 Clinical characteristics of patients based on the genotypes of selected SNPs.

Additional file 2. Supplementary table $\mathbf{2}$ The FPRP and statistical power values of the positive results in this study. 
Additional file 3. Supplemental table 3 Haplotype frequencies and the association with the risk of $2 \mathrm{D}$.

\section{Acknowledgements}

We thank all the participants from Hainan General Hospital for providing blood samples and all people involved in this study.

\section{Authors' contributions}

YD and Yunjun Zhang designed this study protocol and drafted the manuscript; XZ and WD performed the DNA extraction and genotyping; JS and ML performed the data analysis; Yutian Zhang and Yunjun Zhang performed the sample collection and information recording. YD conceived and supervised the study. All authors read and approved the final manuscript.

\section{Funding}

This study was supported by Hainan Province Health Industry Research Project (No. 20A200239).

\section{Availability of data and materials}

The datasets generated and/or analysed during the current study are available in the [Zenodo] repository, https://doi.org/10.5281/zenodo.5251076.

\section{Declarations}

\section{Ethics approval and consent to participate}

This study was conducted under the standard approved by the Ethics Committee of Hainan General Hospital, and conformed to the ethical principles for medical research involving humans of the World Medical Association Declaration of Helsinki. All participants signed informed consent forms before participating in this study.

\section{Consent for publication}

Not applicable.

\section{Competing interests}

The authors declared that they have no conflicts of interest.

\section{Author details}

'Department of General Practice, Hainan General Hospital, \#19, Xiuhua Road, Xiuying District, Haikou 570311, Hainan, People's Republic of China. ${ }^{2}$ Health Center, Hainan General Hospital, Haikou 570311, Hainan, People's Republic of China.

Received: 17 May 2021 Accepted: 11 October 2021 Published online: 30 October 2021

\section{References}

1. Laakso M. Biomarkers for type 2 diabetes. Mol Metab. 2019;27S(Suppl):S139-46

2. Langenberg C, Lotta LA. Genomic insights into the causes of type 2 diabetes. Lancet. 2018;391(10138):2463-74.

3. Tremblay J, Hamet P. Environmental and genetic contributions to diabetes. Metab Clin Exp. 2019;100s:153952.

4. Sirdah MM, Reading NS. Genetic predisposition in type 2 diabetes: a promising approach toward a personalized management of diabetes. Clin Genet. 2020;98(6):525-47.

5. Thomas F, Balkau B, Vauzelle-Kervroedan F, Papoz L. Maternal effect and familial aggregation in NIDDM. The CODIAB Study. CODIAB-INSERMZENECA Study Group. Diabetes. 1994;43(1):63-7.

6. Poulsen P, Grunnet LG, Pilgaard K, Storgaard H, Alibegovic A, Sonne MP, et al. Increased risk of type 2 diabetes in elderly twins. Diabetes. 2009;58(6):1350-5.

7. Sanghera DK, Blackett PR. Type 2 diabetes genetics: beyond GWAS. J Diabetes Metab. 2012;3(198):6948.

8. Cole JB, Florez JC. Genetics of diabetes mellitus and diabetes complications. Nat Rev Nephrol. 2020;16(7):377-90.
9. Sladek R, Rocheleau G, Rung J, Dina C, Shen L, Serre D, et al. A genomewide association study identifies novel risk loci for type 2 diabetes. Nature. 2007:445(7130):881-5

10. Frayling TM, Timpson NJ, Weedon MN, Zeggini E, Freathy RM, Lindgren $\mathrm{CM}$, et al. A common variant in the FTO gene is associated with body mass index and predisposes to childhood and adult obesity. Science. 2007;316(5826):889-94.

11. Scott LJ, Mohlke KL, Bonnycastle LL, Willer CJ, Li Y, Duren WL, et al. A genome-wide association study of type 2 diabetes in Finns detects multiple susceptibility variants. Science. 2007;316(5829):1341-5.

12. Saxena R, Voight BF, Lyssenko V, Burtt NP, de Bakker PI, Chen H, et al. Genome-wide association analysis identifies loci for type 2 diabetes and triglyceride levels. Science. 2007;316(5829):1331-6.

13. Steinthorsdottir $V$, Thorleifsson $G$, Reynisdottir I, Benediktsson $R$, Jonsdottir T, Walters GB, et al. A variant in CDKAL1 influences insulin response and risk of type 2 diabetes. Nat Genet. 2007;39(6):770-5.

14. Vujkovic M, Keaton JM, Lynch JA. Discovery of 318 new risk loci for type 2 diabetes and related vascular outcomes among 1.4 million participants in a multi-ancestry meta-analysis. Nat Gentics. 2020;52(7):680-91.

15. Mashal S, Khanfar M, Al-Khalayfa S, Srour L, Mustafa L, Hakooz NM, et al. SLC30A8 gene polymorphism rs13266634 associated with increased risk for developing type 2 diabetes mellitus in Jordanian population. Gene. 2021;768:145279.

16. Mahajan A, Taliun D, Thurner M, Robertson NR, Torres JM, Rayner NW, et al. Fine-mapping type 2 diabetes loci to single-variant resolution using high-density imputation and islet-specific epigenome maps. Nat Genetics. 2018;50(11):1505-13.

17. Bacchelli E, Ceroni F, Pinto D, Lomartire S, Giannandrea M, D'Adamo P, et al. A CTNNA3 compound heterozygous deletion implicates a role for aT-catenin in susceptibility to autism spectrum disorder. J Neurodev Disord. 2014:6(1):17.

18. Chiarella SE, Rabin EE. aT-catenin: a developmentally dispensable, disease-linked member of the a-catenin family. Tissue Barriers. 2018;6(2):e1463896

19. Eckel RH, Grundy SM, Zimmet PZ. The metabolic syndrome. Lancet. 2005;365(9468):1415-28.

20. Tekola-Ayele F, Doumatey AP, Shriner D, Bentley AR, Chen G, Zhou J, et al. Genome-wide association study identifies African-ancestry specific variants for metabolic syndrome. Mol Genet Metab. 2015;116(4):305-13.

21. Cullmann M, Hilding A, Östenson CG. Alcohol consumption and risk of pre-diabetes and type 2 diabetes development in a Swedish population. Diabet Med. 2012:29(4):441-52.

22. Manson JE, Ajani UA, Liu S, Nathan DM, Hennekens CH. A prospective study of cigarette smoking and the incidence of diabetes mellitus among US male physicians. Am J Med. 2000;109(7):538-42.

23. Wu Y, Ding Y, Tanaka Y, Zhang W. Risk factors contributing to type 2 diabetes and recent advances in the treatment and prevention. Int J Med Sci. 2014;11(11):1185-200.

24. He J, Zou Y, Liu X, Zhu J, Zhang J, Zhang R, et al. Association of common genetic variants in pre-microRNAs and neuroblastoma susceptibility: a two-center study in Chinese children. Mol Ther Nucleic Acids. 2018;11:1-8.

25. Wang $X$, Bao W, Liu J, Ouyang YY, Wang D, Rong S, et al. Inflammatory markers and risk of type 2 diabetes: a systematic review and meta-analysis. Diabetes Care. 2013;36(1):166-75.

26. Malik VS, Popkin BM, Bray GA, Després JP, Willett WC, Hu FB. Sugar-sweetened beverages and risk of metabolic syndrome and type 2 diabetes: a meta-analysis. Diabetes Care. 2010;33(11):2477-83.

27. Imamura F, O'Connor L, Ye Z, Mursu J, Hayashino Y, Bhupathiraju SN, et al. Consumption of sugar sweetened beverages, artificially sweetened beverages, and fruit juice and incidence of type 2 diabetes: systematic review, meta-analysis, and estimation of population attributable fraction. BMJ. 2015;351:h3576.

28. Hemmingsen B, Gimenez-Perez G, Mauricio D, Roqué IFM, Metzendorf MI Richter B. Diet, physical activity or both for prevention or delay of type 2 diabetes mellitus and its associated complications in people at increased risk of developing type 2 diabetes mellitus. Cochrane Database Syst Rev. 2017:12(12):Cd003054.

29. Basu S, Yoffe P, Hills N, Lustig RH. The relationship of sugar to populationlevel diabetes prevalence: an econometric analysis of repeated crosssectional data. PLoS ONE. 2013:8(2):e57873. 
30. Kautzky-Willer A, Harreiter J, Pacini G. Sex and gender differences in risk, pathophysiology and complications of type 2 diabetes mellitus. Endocr Rev. 2016;37(3):278-316.

31. Bray GA. Medical consequences of obesity. J Clin Endocrinol Metab. 2004;89(6):2583-9.

32. Fraser A, Harris R, Sattar N, Ebrahim S, Davey Smith G, Lawlor DA. Alanine aminotransferase, gamma-glutamyltransferase, and incident diabetes: the British Women's Heart and Health Study and meta-analysis. Diabetes Care. 2009:32(4):741-50.

33. Hafner J, Zadrazil M, Grisold A, Ricken G, Krenn M, Kitzmantl D, et al. Retinal and corneal neurodegeneration and their association with systemic signs of peripheral neuropathy in type 2 diabetes. Am J Ophthalmol. 2020:209:197-205.
34. Kunutsor SK, Abbasi A, Apekey TA. Aspartate aminotransferase-risk marker for type-2 diabetes mellitus or red herring? Front Endocrinol (Lausanne). 2014;5:189.

35. Schneider AL, Lazo M, Ndumele CE, Pankow JS, Coresh J, Clark JM, et al. Liver enzymes, race, gender and diabetes risk: the Atherosclerosis Risk in Communities (ARIC) Study. Diabet Med. 2013;30(8):926-33.

\section{Publisher's Note}

Springer Nature remains neutral with regard to jurisdictional claims in published maps and institutional affiliations.
Ready to submit your research? Choose BMC and benefit from:

- fast, convenient online submission

- thorough peer review by experienced researchers in your field

- rapid publication on acceptance

- support for research data, including large and complex data types

- gold Open Access which fosters wider collaboration and increased citations

- maximum visibility for your research: over $100 \mathrm{M}$ website views per year

At BMC, research is always in progress.

Learn more biomedcentral.com/submissions 